\title{
Highlights
}

- BPA promotes macrophage polarization toward proinflammatory M1 subtype in vitro.

- BPA inhibits macrophage toward anti-inflammatory M2 subtype polarization in vitro.

- BPA enhances pro-inflammatory cytokines secretion while reduces anti-inflammatory cytokines production.

- BPA promotes macrophage polarization toward proinflammatory M1 subtype associated with upregulated expression of IRF5. 


\section{Bisphenol A promotes macrophage proinflammatory subtype polarization via upregulation of IRF5 expression in Vitro}

Xiaotong $\mathrm{Lu}^{\text {a\#}}$, Meiling $\mathrm{Li}^{\mathrm{b} \# \&}$, Changhao $\mathrm{Wu}^{\mathrm{c}}$,Chengfan Zhou ${ }^{\mathrm{a}}$, Jiaxiang Zhang ${ }^{\mathrm{a}}$, Qixing Zhu ${ }^{\mathrm{a}, \mathrm{d}^{*}}$, Tong Shen ${ }^{\mathrm{a}, \mathrm{b}, \mathrm{d}^{*}}$

a Department of Occupational Health and Environmental Health, School of Public Health, Anhui Medical University, Hefei 230032, Anhui, PR China

bDepartment of Toxicology, School of Public Health, Anhui Medical University, Hefei 230032, Anhui, PR China

${ }^{\mathrm{c}}$ Department of Biochemistry and Physiology, Faculty of Heath \& Medical Sciences, University of Surrey, Surrey, Guildford, UK

d Institute of Dermatology, Anhui Medical University, Hefei 230032, Anhui, PR China

\# These authors contributed equally to this work.

\&Meiling Li present address: Department of Chronic Diseases, Center for Disease Control and Prevention of Yuhuatai District, Nanjing City 210012, Jiangsu Province, PR China

*Co-Corresponding author:

Prof. Tong Shen, Department of Occupational Health and Environmental Health, School of Public Health, Anhui Medical University, Hefei 230032, Anhui, PR China. Phone: +86 055165169772, fax: +86 0551 63869179, E-mail: shentong@ahmu.edu.cn or ahmusht@163.com Prof. Qixing Zhu,Institute of Dermatology, Anhui Medical University, Hefei 230032, Anhui, PR China; Department of Occupational Health and Environmental Health, School of Public Health, Anhui Medical University, Hefei 230032, Anhui, PR China.

Phone: +86055162923009 E-mail: zqxing@yeah.net

\section{Runing Title}

BPA promotes macrophage proinflammatory polarization 


\title{
Bisphenol A promotes macrophage proinflammatory subtype polarization via upregulation of IRF5 expression in Vitro
}

Xiaotong $\mathrm{Lu}^{\mathrm{a} \#}$ Meiling $\mathrm{Li}^{\mathrm{b} \# \&}$, Changhao $\mathrm{Wu}^{\mathrm{c}}$, Chengfan Zhou ${ }^{\mathrm{a}}$, Jiaxiang Zhang ${ }^{\mathrm{a}}$, Qixing Zhu ${ }^{\mathrm{a}, \mathrm{d}^{*}}$, Tong Shen ${ }^{\mathrm{a}, \mathrm{b}, \mathrm{d}^{*}}$

${ }^{\text {a }}$ Department of Occupational Health and Environmental Health, School of Public Health, Anhui Medical

University, Hefei 230032, Anhui, PR China

bDepartment of Toxicology, School of Public Health, Anhui Medical University, Hefei 230032, Anhui, PR China

${ }^{\mathrm{c}}$ Department of Biochemistry and Physiology, Faculty of Heath \& Medical Sciences, University of Surrey,

Surrey, Guildford, UK

${ }^{\mathrm{d}}$ Institute of Dermatology, Anhui Medical University, Hefei 230032, Anhui, PR China

\# These authors contributed equally to this work.

\&Meiling Li present address: Department of Chronic Diseases, Center for Disease Control and Prevention of Yuhuatai District, Nanjing City 210012, Jiangsu Province, PR China

*Co-Corresponding author: Prof. Tong Shen, E-mail: shentong@ahmu.edu.cn or ahmusht@163.com; Prof. Qixing Zhu, E-mail: zqxing@yeah.net

\begin{abstract}
Exposure to environmental endocrine-disrupting chemical Bisphenol-A (BPA) is closely associated with an imbalance of immune homeostasis, but the underlying mechanisms are not fully understood. In the present study, the effects of BPA on the polarization of mouse peritoneal macrophages were investigated in vitro. Environmentally relevant low concentrations of BPA treatment under M1 type polarization conditions increased the number of M1 subtype macrophages, the gene expression of M1 phenotypic marker CD11c and the activity and gene expression of M1 functional marker iNOS, as well as the production of pro-inflammatory cytokines. However, The same dose BPA treatment under M2 type polarization conditions reduced the number of M2 subtype macrophages, the gene expression of M2 phenotypic marker CD206 and the activity and gene expression of M2 functional marker Arg-1, along with the production of anti-inflammatory cytokines. We also identified that the expression of transcription factor IRF5 was upregulated by BPA exposure in M1 macrophages under M1 type polarization conditions. Our results demonstrate that BPA promotes macrophage polarization toward proinflammatory M1 subtype and M1 activity, associated with upregulated expression of IRF5, while BPA inhibits macrophage toward anti-inflammatory M2 subtype polarization. These findings provide new insight into the link between exposure to BPA and impairment of immune functions.
\end{abstract}


Key Words Bisphenol A; polarization; macrophages; transcription factor; inflammation 


\section{Introduction}

Bisphenol A (BPA), one of the highest-production chemicals, is used widely as a monomer in the manufacture of polycarbonate plastics and epoxy resins for daily applications, including food packaging material, water bottle, medical devices, thermal papers, and other materials. Geens et al. estimated the levels of human exposure to BPA to be in the range of $0.1-5 \mu \mathrm{g} / \mathrm{kg} \mathrm{BW} / \mathrm{d}$ from the dietary and nondietary route (Geens et al. 2012). Population data show that BPA can be detected in multiple biological samples from more than 90\% of humans (Mustieles et al., 2019; LaKind and Naiman, 2015; Vandenberg et al., 2010). Increasing evidence reveals that human exposure to low concentration BPA is closely associated with inflammation and impairment of immune functions (Li et al., 2018; Malaisé et al., 2018; Rogers et al., 2017; Zbucka-Kretowska et al., 2017; Luo et al.,2016; Gostner et al,, 2015; Rogers et al., 2013). Up to now, the exact mechanism of immunotoxicity induced by BPA exposure is not fully understood.

Macrophages are a heterogeneous population of immune cells. They play an essential role in innate and adaptive immunity in response to xenobiotics and mediate the inflammatory process through secreting mediators and cytokines, as well as interacting with other immune cells (Acaroz et al., 2019; Davies et al., 2013; Wynn et al., 2013; McNelis and Olefsky, 2014). The function of macrophages is regulated by their polarization status depending on phenotypic heterogeneity (Gordon et al., 2014; McWhorter et al., 2013). In response to environmental signals, macrophages are capable of polarizing toward two different phenotypes, classically activated M1 subtype and alternatively activated M2 subtype. M1 subtype, also known as the proinflammatory subtype, is characterized by high expression of the membrane marker CD11c and secretion of inducible nitric oxide synthase (iNOS) and proinflammatory cytokines such as tumor necrosis factor (TNF)- $\alpha$, interleukin (IL)-6 and monocyte chemoattractant protein (MCP)-1. In contrast, M2 subtype has the characteristic of relatively high expression of surface marker CD206 and production of arginase (Arg-1) and anti-inflammatory cytokines such as transforming growth factor (TGF)- $\beta$ and IL-10 (Martinez et al., 2009; Sica and Marques, 2012; Murray et al., 2014). Numerous studies have demonstrated BPA exposure-induced downregulation of macrophage activity, decrease in macrophages phagocytic function and influence of inflammatory responses via modulating the cytokine expression of macrophages (Ampem et al., 2019; Berntsen et al., 2018; Li et al., 2018a, 2018b; Teixeria et al., 2016; Liu et al., 2014; Pyo et al., 2007; Byun et al., 2005). However, the 
effects of BPA exposure on macrophage polarization and the underlying molecular mechanisms remain largely undefined.

In the present study, in order to investigate the effects of BPA exposure on polarization of mouse peritoneal macrophages in vitro, we evaluated the alterations in the cell number and the gene expression of phenotype markers CD11c (for M1 subtype) and CD206 (for M2 subtype), the activity and gene expression of functional marker iNOS (for M1 subtype) and Arg-1 (for M2 subtype), and the content and mRNA level of cytokines produced by M1 or M2 subtype after BPA treatment under M1 or M2 type conditions of polarization. Furthermore, the expression of transcription factors interferon regulatory factor (IRF) 5 and IRF4 that modulate the polarization of M1 and M2 subtypes were also investigated. Here, we show that BPA exposure can boost the mouse peritoneal macrophages toward M1 subtype polarization and this is associated with upregulated expression of IRF5, while it inhibits macrophages toward M2 subtype polarization.

\section{Materials and methods}

\subsection{Reagents and Antibodies}

BPA (purity $>99 \%$ ) was purchased from Tianjin Damao Chemical Reagent Factory (Tianjin, China). IFN- $\gamma$ and IL-4 were from Peprotech (Rocky Hill, NJ, USA). Lipopolysaccharide (LPS) from Escherichia Coli (serotype: 055: B5) and Dimethylsulfoxide (DMSO) were obtained from Sigma- Aldrich (St. Louis, USA). Dulbecco's modified eagle medium (DMEM) and fetal bovine serum (FBS) were purchased from Hyclone (Logan, USA). Fluorescein isothiocyanate-conjugated anti-mouse CD11c (FITC-CD11c), FITC-conjugated anti-mouse CD206 (FITC-CD206) and phycoerythrin-conjugated anti-mouse F4/80 (PE-F4/80) were purchased from Biolegend (San Diego, USA). ELISA Kits for quantitative measurement of iNOS, TNF- $\alpha$, IL-6, MCP-1, Arg-1, IL-10 and TGF- $\beta$ were purchased from Calvin Biological Technology co. (Suzhou, China). RNeasy Mini Kit was purchased from Qiagen (Austin, USA). Transcription First Strand cDNA Synthesis kit and Light Cycler 480 SYBR Green I Master were purchased from Roche (Basel, Switzerland). PCR-Primers were obtained from Takara BIO (Dalian, China). Anti-mouse IRF4, anti-mouse IRF5, and $\beta$-actin antibody were from Abcam (Cambridge, USA). BPA was dissolved in DMSO (the final concentration of DMSO in the medium was no more than $0.1 \%$ ).

2.2 Isolation of peritoneal macrophages from mice 
The eight-week-old specific pathogen free (SPF) and healthy C57BL/6J mice were bought from Laboratory Animal Center of Anhui Province and anesthetized with chloral hydrate. 5 ml DMEM containing $100 \mathrm{U} / \mathrm{ml}$ penicillin and $100 \mu \mathrm{g} / \mathrm{ml}$ streptomycin were injected intraperitoneally, peritoneal lavage fluid was extracted after $5 \mathrm{~min}$ and then followed by centrifugation for $8 \mathrm{~min}$ at $1000 \mathrm{rpm}$. The pellet was resuspended in DMEM and the cell suspension was adjusted to $2 \times 10^{6} /$ $\mathrm{mL}$, seeded in 12-well culture plates and incubated for $3 \mathrm{~h}$ under $5 \% \mathrm{CO}_{2}$ at $37^{\circ} \mathrm{C}$. Then the medium was replaced with DMEM supplemented with $100 \mathrm{U} / \mathrm{mL}$ penicillin, $100 \mu \mathrm{g} / \mathrm{mL}$ streptomycin and 10\% heat-inactivated FBS. The experiment was approved by the ethics committee of Anhui Medical University.

2.3 Macrophage polarization toward M1 phenotype or M2 phenotype and treatment with BPA

For M1 polarization experiments, peritoneal macrophage was treated with $10 \mathrm{ng} / \mathrm{ml} \mathrm{IFN-} \gamma$ for $24 \mathrm{~h}$ and then stimulated with $500 \mathrm{ng} / \mathrm{ml}$ LPS for an additional $24 \mathrm{~h}$ (M1 type conditions). For M2 polarization experiments, macrophage was treated with $10 \mathrm{ng} / \mathrm{ml}$ IL-4 for $24 \mathrm{~h}$ (M2 type conditions). To evaluate the results of macrophage polarization in M1 or M2 type conditions of polarization, cell morphology were observed and ananlyzed, the number of M1 phenotype or M2 phenotype macrophages were analyzed by phenotypic markers CD11c or CD206 using flow cytometry; activities of functional marker iNOS or Arg-1 in the culture medium were measured using ELISA assay.

To explore the effects of BPA exposure on macrophage polarization, the cells were treated with various concentrations of BPA $(0.1,1$ and $10 \mu \mathrm{M})$ throughout the polarization process. $0.1 \%$ DMSO was used as vehicle control.

\subsection{Analysis of cell morphology}

Cell morphology were observed using a Leica DMI1 inverted microscope. The images of cells were captured, the cell area and the elongation factor were analyzed by Image-pro plus software V6.0 (Media Cybernetics, Inc.) as previously described (McWhorter et al., 2013). The elongation factor was defined as the longest axis length divided by the short axis (across the cell nucleus) length. Cell area, the long axis and the short axis of each cell were analyzed in the same unit area.

\subsection{Flow cytometry analysis}

Subtypic analysis of polarizated macrophage was performed using flow cytometry. Following polarization, cells were recovered from culture plates by scrapping, rinsed in phosphate-buffered 
saline (PBS) and centrifuged twice at $1500 \mathrm{rpm}$ for $5 \mathrm{~min}$. Subsequently, cells were adjusted to $5 \times 10^{5} \mathrm{cells} / \mathrm{ml}$ and incubated with $10 \%$ FBS for 20 min at $4{ }^{\circ} \mathrm{C}$ to block nonspecific binding. The cells were then stained for $30 \mathrm{~min}$ in the dark at $4{ }^{\circ} \mathrm{C}$ with FITC-CD11c and PE- F4/80 for analysis of M1 phenotype under M1 type conditions, or with FITC-CD206 and PE- F4/80 for analysis of M2 phenotype under M2 type conditions. Isotypic controls were used in all cases. After staining, the cells were fixed with $2 \% \mathrm{w} / \mathrm{v}$ formaldehyde and analyzed immediately with a FACScalibur flow cytometer (Beckman Coulter) using Cell Quest 3.1 software.

2.5ㅁ Quantitative real-time polymerase chain reaction (qRT-PCR) analysis

Total RNA was extracted from macrophages by RNeasy Mini Kit, and then cDNA was synthesized using Transcription First Strand cDNA Synthesis kit. For real-time PCR, cDNA was amplified on the LightCycler® 480 Real-Time PCR system (Roche LifeScience) using Light Cycler 480 SYBR Green I Master. The PCR program was as follows: pre-degeneration at $95^{\circ} \mathrm{C}$ for $5 \mathrm{~min}$, the next, 45 cycles of $15 \mathrm{sec}$ at $95^{\circ} \mathrm{C}$ for denaturing and $15 \mathrm{sec}$ at $60^{\circ} \mathrm{C}$ for annealing, and finally $30 \mathrm{sec}$ at $72^{\circ} \mathrm{C}$ for extension. The relative quantity of mRNA was calculated using the standard $2^{-\Delta \Delta C t}$ method with $\beta$-actin serving as an internal standard. All fold changes are normalized to the vehicle control. Gene-specific primers were as follows:

\begin{tabular}{lll}
\hline Gene & Forward primer & Reverse primer \\
\hline CD11c & GAGCCAGAACTTCCCAACTG & TCAGGAACACGATGTCTTGG \\
CD206 & CAGGTGTGGGCTCAGGTAGT & TGTGGTGAGCTGAAAGGTGA \\
iNOS & CAGCTGGGCTGTACAAACCTT & CATTGGAAGTGAAGCGTTTCG \\
Arg-1 & CTCCAAGCCAAAGTCCTTAGAG & AGGAGCTGTCATTAGGGACATC \\
TNF- $\alpha$ & AAG GCC GGG GTG TCC TGG AG & AGG CCA GGT GGG GAC AGC TC \\
IL-6 & CCACTTCACAAGTCGGAGGCTTA & GCAAGTGCATCATCGTTGTTCATAC \\
MCP-1 & TCCCAATGAGTAGGCTGGAG & AAGTGCTTGAGGTGGTTGTG \\
IL-10 & CCAAGCCTTATCGGAAATGA & TTCACAGGGGAGAAATCG \\
TGF- $\beta$ & TGG TGG ACC GCA ACA ACG CC & GGG GGT TCG GGC ACT GCT TC \\
IRF4 & CTTGCAAGCTCTTTGACACACAG & GAAACTCCTCACCAAAGCACAG \\
IRF5 & ATGGGGACAACACCATCTTC & CAGGTTGGCCTTCCACTTG \\
$\beta$-actin & CGCCCTAGGCACCAGGGTGTG & TCGGTGAGCAGCACAGGGTG \\
\hline
\end{tabular}

2.67 Enzyme-linked immunosorbent assay (ELISA) assays 
Cell-free supernatants were harvested after polarization. Activities of iNOS and Arg-1 and the contents of TNF- $\alpha$, IL-6, MCP-1, TGF- $\beta$ and IL-10 in the supernatants were quantified by ELISA Kits, according to the manufacturer's instructions.

\section{$2.7 \underline{8}$ Western blot analysis}

Cells were lysed with ice-cold Tris buffer (50 mM Tris-HCl pH 6.8, 2\% SDS, 10\% glycerol, 2.5\% $\beta$-mercaptoethanol) containing protease inhibitor cocktail. The supernatant was collected, and the concentration of lysate protein was determined using a Nanodrop 2000 (Thermo Scientific, Holtsville, USA). Equal amounts of protein $(20 \mu \mathrm{g})$ were mixed with sample buffer, electrophoresed through $12.5 \%$ SDS polyacrylamide gel (PAGE) and then transferred to polyvinylidene fluoride (PVDF) membranes. The membranes were blocked with 5\% nonfat milk for $1 \mathrm{~h}$ and then incubated with the primary antibody ( anti-mouse IRF4 or anti-mouse IRF5, 1:2000 ) at $4^{\circ} \mathrm{C}$ overnight. Thereafter, the membranes were incubated with the secondary antibody, horseradish peroxidase (HRP) labeled goat anti-rabbit $\operatorname{IgG}(1: 5000)$ at room temperature for $2 \mathrm{~h}$. Results were visualized with enhanced chemiluminescence (ECL) kit (Pierce Biotech). $\beta$ actin antibody was used as the loading control. Images were analyzed by ImageJ software.

\subsection{Statistical analysis}

All independent experiments were repeated at least three times $(n=3)$. Data are presented as means \pm S.E.M. unless otherwise stated. Statistical analyses were performed using GraphPad Prism 5 (GraphPad Software, Inc. La Jolla, CA, USA). Comparisons among multiple groups were performed using one-way analysis of variance (ANOVA) followed by Tukey post hoc test. A $p$ value of less than 0.05 was considered statistically significant.

\section{Results}

3.1 Macrophages polarize toward M1 subtype under M1 type conditions, and toward M2 subtype under M2 type conditions

Compared with unpolarized macrophages (Fig.1A), we observed dramatic alterations in the cell shape: most of the cells became rounder after polarization under M1 type conditions (Fig.1B), while exhibited an elongated fibroblast-like shape after polarization under M2 type conditions (Fig.1C). The cell area wasn't marked difference between cell after polarization under M1 type conditions and unpolarized counterparts, but there was significantly higher in cell after 
polarization under M2 type conditions than that of unpolarized counterparts (Fig. 1D). The elongation factor of cell was significantly lower in cell after polarization under M1 type conditions than that of unpolarized counterparts, however there was significantly higher in cell after polarization under M2 type conditions than that of unpolarized counterparts (Fig. 1E). These shape changes are similar to those from previous publications (McWhorter et al., 2013). The number of M1 phenotype cells and activity of iNOS were significantly increased by 2.50 and 5.26 times respectively compared with those from the unpolarized counterparts after polarization under M1 type conditions. The number of M2 phenotype cells and activity of Arg-1 were also significantly increased by 2.33 and 7.96 folds respectively compared with those in unpolarized counterparts after polarization under M2 type conditions (Table 1). These results show that unpolarized macrophages can polarize toward M1 subtype under M1 type conditions, while they can polarize toward M2 subtype under M2 type conditions.

3.2 BPA promotes macrophages toward M1 subtype polarization, but inhibits them toward M2 subtype polarization

To investigate the effects of BPA exposure on macrophage polarization, the numbers of M1 or M2 subtype cells, the gene expressions of M1 or M2 phenotypic markers, the activities and mRNA expressions of M1 or M2 functional markers were measured. The number of CD11c positive cells was significantly increased in BPA exposure groups compared to that in the controls under M1 type conditions (Fig.2 A and B). The expression of M1 phenotypic marker CD11c mRNA was also markedly enhanced by BPA exposure under M1 type conditions (Fig.2 C). Along with both the activity and mRNA expression of M1 subtype, the functional marker iNOS was remarkably elevated in BPA exposure groups under M1 type conditions (Fig.2 D and E). In contrast to promoting M1 subtype polarization, BPA treatment significantly reduced the number of CD206 positive cells (Fig. $3 \mathrm{~A}$ and B) and inhibited the mRNA expression of M2 phenotypic marker CD206(Fig.3 C), in combination with reduced activity and gene expression of M2 subtype functional marker Arg-1(Fig.3 D and E), depending on the dose under M2 type conditions. These results suggest that BPA exposure promotes mouse peritoneal macrophage toward M1 subtype polarization but inhibits M2 subtype polarization.

3.3 BPA induces the production of cytokines associated with M1 subtype, whereas suppresses the production of cytokines associated with M2 subtype 
To explore if exposure to low dose BPA also affects the function of the different macrophage subtypes, we next examined the production of cytokines associated with M1 or M2 subtype macrophages. Treatment with $0.1,1$ and $10 \mu \mathrm{M}$ of BPA, not only raised the content in supernatants but also enhanced the mRNA expression of proinflammatory cytokines, including TNF- $\alpha$, IL-6, MCP-1, under M1 type conditions (Fig.4). On the other hand, the content and mRNA expression of anti-inflammatory cytokines IL-10 and TGF- $\beta$ were decreased under M2 type conditions (Fig.5). These results indicate that BPA exposure induces the production of cytokines associated with M1 subtype, whereas it inhibits the production of cytokines associated with M2 subtype. 3.4 BPA upregulates the expression of IRF5, but does not affect the expression of IRF4 under M1 type conditions

To better understand the underlying molecular mechanisms of BPA exposure-boosted macrophage polarization toward M1 subtype under M1 type conditions, we further investigated the expression of transcription factor IRF5 and IRF4 at mRNA and protein levels. As shown in Fig.6, under M1 type conditions, treatment of macrophages with various doses of BPA resulted in upregulated expression of IRF5 at both mRNA level and protein level, with significant difference among different dose groups. However, there was no detectable effect on the expression of IRF4 at either mRNA level or protein level. These results indicate that upregulated expression of IRF5 was involved in macrophage polarization toward M1 subtype induced by BPA exposure under M1 type conditions, independent from IRF4.

3.5 BPA has no effect on the expression of IRF5 and IRF4 under M2 type conditions We sought to identify if IRF5 and IRF4 were implicated in the inhibition of macrophage polarization toward M2 subtype induced by exposure to BPA under M2 type conditions and measured the expression of IRF5 and IRF4 at mRNA and protein levels. As shown in Fig.7, there was no significant difference in the expression of IRF5 and IRF4 at either mRNA level or protein level after BPA treatment under M2 type conditions. These results demonstrate that IRF5 and IRF4 are not involved in the inhibition of macrophage polarization toward M2 subtype induced by BPA exposure under M2 type conditions.

\section{Discussion}

The biological effects induced by exposure to environmentally relevant concentrations of BPA 
have attracted increasing interest (Wassenaar, et al., 2017). European Food Safety Authority (EFSA) has lowered the tolerable daily intake level (TDI) of BPA from $50 \mu \mathrm{g} / \mathrm{kg} \mathrm{BW} / \mathrm{d}$ to a preliminary TDI of $4 \mu \mathrm{g} / \mathrm{kg} \mathrm{BW} / \mathrm{d}$ in January 2015 (EFSA, 2015). A number of studies demonstrated that micromolar range of BPA could induce various effects in vitro or in vivo (Kim et al., 2018; Senylidiz et al., 2017; Pfeifer et al., 2015). A recent study reported that DNA damage and proliferative effects induced by BPA were only significant at concentrations less than $10 \mu \mathrm{M}$ in 2D HepG2 cultures (Kim et al., 2018), suggesting a non-linear dose response relationship. Most importantly, the immune system is especially sensitive to BPA exposure (Li et al., 2018(a); Li et al., 2018(b)). In the present study, we evaluated the effects of micromolar concentrations BPA on macrophage polarization and explored the underlying mechanisms.

Macrophages have been recognized as important effectors and regulators of inflammation and the immune response, and their function depends on the phenotypes. The phenotypic polarization of macrophages is mediated by a milieu of cues in the local microenvironment and leads to dramatic alterations in the expression of the surface and functional markers (Lumeng et al., 2007). Here, we demonstrate that mouse peritoneal macrophages are susceptible to BPA at levels relevant to environmental exposure; moreover, exposure to BPA at the concentration more than $1 \mu \mathrm{M}$ can significantly promote polarization toward M1 subtype, shown as high expression of the surface marker CD11c and functional marker iNOS. Meanwhile, the same concentrations of BPA exposure also significantly inhibit macrophage polarization toward M2 subtype, manifest as the low expression of the surface marker CD206 and functional marker Arg-1.

A change of macrophage phenotype can give rise to distinct functions that are phenotypically characterized by the production of proinflammatory and anti-inflammatory cytokines, which provides an important tool for understanding the regulation of the inflammatory process and immune response (Kraakman et al., 2014; Chatterjee et al., 2013; Kamei et al., 2006; Kanda et al., 2006) . M1 subtype macrophage produces proinflammatory cytokines thus contributing to inflammation and T-helper (Th)1-type immune response, while M2 subtype macrophage secrets anti-inflammatory cytokines involved in inflammation resolution and Th2-type immune response (Ip et al., 2017; Sica et al., 2012; Martinez et al., 2009). In the present study, exposure to low dose BPA stimulates the expression of proinflammatory cytokines including TNF- $\alpha$, IL-6, MCP-1 and inhibits the expression of anti-inflammatory cytokines IL-10 and TGF- $\beta$. These results are in 
accordance with previous publications in that direct BPA exposure or perinatal BPA exposure affect inflammatory cytokine release from peritoneal macrophages (Li et al., 2018) or gut tissue (Malaisé et al., 2018). However, the earlier study which tested peritoneal macrophages used higher concentrations of BPA and did not differentiate macrophage subtypes (Li et al., 2018). The present study used lower concentrations of BPA and identified macrophage subtype-specific effects.

Furthermore, these changes in cytokine production by M1 subtype or M2 subtype macrophages are coincided with the change in the number of M1 subtype or M2 subtype macrophages. Thus, a shift of M1 and M2 balance towards M1 predominance would promote a proinflammatory reaction. Therefore, we propose that BPA exposure-induced upregulation of M1 subtype polarization and downregulation of M2 subtype polarization and corresponding changes in the release of their respective inflammatory cytokines may be involved in altering immune responses and play a proinflammation role in inflammatory disorders, such as obesity, type 2 diabetes and tumor.

Transcription factor interferon regulatory factors (IRFs) play diverse functional roles in the transcriptional regulation of the immune system (Lawrence and Natoli, 2011; Krausgruber et al., 2011; Yanai et al., 2007) . Previous studies have identified the IRF5 as the master regulator of the M1 macrophage polarization. Furthermore, IRF5 dictates the expression of proinflammatory genes such as IL12 $\beta$ and IL23a whilst repressing anti-inflammatory genes such as IL10 (Weiss et al., 2015; Paun et al., 2008) . IRF4 is a negative regulator of inflammation in diet-induced obesity, in part through regulation of macrophage polarization (Huang et al., 2016; Eguchi et al., 2013; Satoh et al., 2010; Honma et al., 2005). Here we show that murine peritoneal macrophages exposed to BPA are also characterized by high levels of IRF5 mRNA and protein, and express proinflammatory cytokines under M1 type condition, but exhibit no notable change under M2 type condition. These results support the notion that IRF5 contributes to M1 type polarization and enhanced proinflammatory cytokine release in low dose BPA-induced immunological response. However, we did not observe any significant difference in the expression of IRF4 after BPA treatment under either M1 or M2 type conditions, excluding the involvement of IRF4. Thus, these data suggest that IRF5 may contribute not only to BPA induced M1 subtype polarization but also to the secretion of proinflammatory cytokines in macrophages. 
In conclusion, our data demonstrate that submicromolar concentrations of BPA can promote mouse peritoneal macrophage polarization toward M1 subtype and inhibit polarization toward M2 subtype, and this effect is accompanied by enhanced secretion of proinflammatory factors, which appear to be mediated by IRF5 expression in vitro. However, additional research will be needed to confirm our findings regarding the effects of BPA exposure on macrophages polarization and precise molecular mechanisms of regulation in vivo. Our study provides a new insight into the link between exposure to BPA and impairment of immune function.

\section{Conflict of interest}

The authors declare no conflict of interest.

\section{Acknowledgments:}

This research was supported by the National Natural Science Foundation of China (Grant No. 81473015, 81773467), Anhui Provincial Natural Science Foundation (Grant No. 1508085MH161) and National College Students Innovations Project Foundation (Grant No. 20180366029). CW acknowledges the support from Biotechnology and Biological Sciences Research Council [BB/P004695/1] and National Institute of Aging [1R01AG049321-01A1].

\section{References}

Acaroz, U., Ince, S., Arslan-Acaroz, D., Gurler, Z., Demirel, H.H., Kucukkurt, I., Eryavuz, A., Kara, R., Varol, N., Zhu, K.2019. Bisphenol-A induced oxidative stress, inflammatory gene expression, and metabolic and histopathological changes in male Wistar albino rats: protective role of boron. Toxicol. Res. (Camb). 8, 262-269. doi: 10.1039/c8tx00312b.

Ampem, G., Junginger, A., Yu, H., Balogh, L., Thuróczy, J., Schneider, M.E., Röszer, T., 2019. The environmental obesogen bisphenol A increases macrophage self-renewal. Cell Tissue Res. doi: 10.1007/s00441-019-03019-5.

Berntsen, H.F., Bølling, A.K., Bjørklund, C.G., Zimmer, K., Ropstad, E., Zienolddiny, S., Becher, R., Holme, J.A., Dirven, H., Nygaard, U.C., Bodin, J., 2018. Decreased macrophage phagocytic function due to xenobiotic exposures in vitro, difference in sensitivity between various macrophage models. Food Chem. Toxicol. 112, 86-96. doi: 10.1016/j.fct.2017.12.024. 
Byun, J.A., Heo, Y., Kim, Y.O., Pyo, M.Y., 2005. Bisphenol A-induced downregulation of murine macrophage activities in vitro and ex vivo. Environ. Toxicol. Pharmacol. 19,19-24. doi: 10.1016/j.etap.2004.02.006.

Chatterjee, P., Seal, S., Mukherjee, S., Kundu, R., Mukherjee, S., Ray, S., Mukhopadhyay,S., Majumdar, S.S., Bhattacharya, S., 2013. Adipocyte fetuin-A contributes to macrophage migration into adipose tissue and polarization of macrophages. J. Biol. Chem. 288, 2832428330. doi: $10.1074 /$ jbc.C113.495473.

Davies, L.C., Jenkins, S.J., Allen, J.E., Taylor, P.R., 2013. Tissue-resident macrophages. Nat Immunol. 14(10): 986-995. doi: 10.1038/ni.2705.

EFSA (European Food Safety Authority), 2015. Scientific opinion on the risks to public health related to the presence of bisphenolA(BPA) in food stuffs. EFSA J 13, 3978, https://doi.org/10.2903/j.efsa.2015.3978.

Eguchi, J., Kong, X., Tenta, M., Wang, X., Kang, S., Rosen, E.D., 2013. Interferon regulatory factor 4 regulates obesity-induced inflammation through regulation of adipose tissue macrophage polarization. Diabetes. 62,3394-3403. doi: 10.2337/db12-1327.

Geens, T., Aerts, D., Berthot, C., Bourguignon, J.P., Goeyens, L., Lecomte, P., Maghuin-Rogister, G., Pironnet, A.M., Pussemier, L.. 2012. A review of dietary and non-dietary exposure to bisphenol-A. Food Chem. Toxicol. 50, 3725-3740. https://doi.org/10.1016/j.fct.2012.07.059.

Gordon, S., Pluddemann, A., Martinez, Estrada, F., 2014. Macrophage heterogeneity in tissues: phenotypic diversity and functions. Immunol. Rev. 262, 36-55. doi: 10.1111/imr.12223.

Gostner, J.M., Raggl, E., Becker, K., ?berall, F., Schennach, H., Pease, J.E., Fuchs, D., 2015. Bisphenol A suppresses Th1-type immune response in human peripheral blood mononuclear cells in vitro. Immunol. Lett. 168, 285-292. doi: 10.1016/j.imlet.2015.10.006.

Honma, K., Udono, H., Kohno, T., Yamamoto, K., Ogawa, A., Takemori, T., Kumatori, S., Suzuki, T., Matsuyama, Yui, K., 2005. Interferon regulatory factor 4 negatively regulates the production of proinflammatory cytokines by macrophages in response to LPS. Proc Natl Acad Sci U S A. 102, 16001-16006. doi: 10.1073/pnas.0504226102.

Huang, S.C., Smith, A.M., Everts, B., Colonna, M., Pearce, E.L., Schilling, J.D,, Pearce, E.J., 2016. Metabolic reprogramming mediated by the mTORC2-IRF4 signaling axis is essential for macrophage alternative activation. Immunity. 45, 817-830. doi: 10.1016/j.immuni.2016.09.016. 
Ip, W.K.E., Hoshi, N., Shouval, D.S., Snapper, S., 2017. Medzhitov R. Anti-inflammatory effect of IL10 mediated by metabolic reprogramming of macrophages. Science. 356, 513-519. doi: 10.1126/science.aal3535.

Kamei, N., Tobe, K., Suzuki, R., Ohsugi, M., Watanabe, T., Kubota, N., et al, 2006. Overexpression of monocyte chemoattractant protein-1 in adipose tissues causes macrophage recruitment and insulin resistance. J. Biol. Chem. 281,26602-26614. doi: 10.1074/jbc.M601284200.

Kanda, H., Tateya, S., Tamori, Y., Kotani, K., Hiasa, K., Kitazawa, R., S. Kitazawa, H. Miyachi, S. Maeda, K. Egashira, and M. Kasuga, 2006. MCP-1 contributes to macrophage infiltration into adipose tissue, insulin resistance, and hepatic steatosis in obesity. J. Clin. Invest. 116,1494-1505. doi: $10.1172 / \mathrm{JCI} 26498$.

Kim, S., Mun, G., Choi, E., Kim, M., Jeong, S., Kang, K.W., Jee, S., Lim, K.M., Lee, Y.S., 2018. Submicromolar bisphenol A induces proliferation and DNA damage in human hepatocyte cell lines in vitro and in juvenile rats in vivo. Food Chem, Toxicol. 111, 125-132.

Kraakman, M.J., Murphy, A.J., Jandeleit-Dahm, K., Kammoun, H.L., 2014. Macrophage polarization in obesity and type 2 diabetes: weighing down our understanding of macrophage function? Front immunol. 5, 470. doi: 10.3389/fimmu.2014.00470.

Krausgruber, T., Blazek, K., Smallie, T., Alzabin, S., Lockstone, H., Sahgal, N., Hussell, T., Feldmann, M., Udalova, I.A., 2011. IRF5 promotes inflammatory macrophage polarization and TH1-TH17 responses. Nat. Immunol. 12,231-238. doi: 10.1038/ni.1990.

Lakind, J.S., Naiman, D.Q., 2011. Daily intake of bisphenol A and potential sources of exposure: $2005-$ 2006 National Health and Nutrition Examination Survey. J. Expo. Sci. Environ. Epidemiol. 21, 272279. doi: $10.1038 /$ jes.2010.9.

Lawrence, T., Natoli, G., 2011. Transcriptional regulation of macrophage polarization: enabling diversity with identity. Nat. Rev. Immunol. 11,750-761. doi: 10.1038/nri3088.

Li, J., Bach, A., Crawford, R.B., Phadnis-Moghe, A.S., Chen, W., D'Ingillo, S., Kovalova, N., SuarezMartinez, J.E., Zhou, J., 2018a. CLARITY-BPA: Effects of chronic Bisphenol A exposure on the immune system: Part 1 - Quantification of the relative number and proportion of leukocyte populations in the spleen and thymus. Toxicology. 396-397,46-53. doi: 10.1016/j.tox.2018.01.004 Li, J., Bach, A., Crawford, R.B., Phadnis-Moghe, A.S., Chen, W., D'Ingillo, S., Kovalova, N., SuarezMartinez, J.E., Zhou, J., 2018b. CLARITY-BPA: Effects of chronic bisphenol A exposure on the 
immune system: Part 2 - Characterization of lymphoproliferative and immune effector responses by splenic leukocytes. Toxicology. 396-397,54-67. doi: 10.1016/j.tox.2018.02.004.

Li, Q., Lawrence, C.R., Nowak, R.A., Flaws, J.A., Bagchi, M.K., Bagchi, I.C., 2018. Bisphenol A and Phthalates modulate peritoneal macrophage function in female mice involving SYMD2-H3K36 dimethylation. Endocrinology. 159, 2216-2228. doi: 10.1210/en.2017-03000.

Liu, Y., Mei, C., Liu, H., Wang, H., Zeng, G., Lin, J., Xu, M., 2014. Modulation of cytokine expression in human macrophages by endocrine-disrupting chemical Bisphenol-A. Biochem Biophys Res Commun. 451,592-598. doi: 10.1016/j.bbrc.2014.08.031.

Lumeng, C.N., Bodzin, J.L., Saltiel, A.R., 2007. Obesity induces a phenotypic switch in adipose tissue macrophage polarization. J. Clin. Invest. 117,175-184. doi: 10.1172/JCI29881.

Luo, S., Li, Y., Li, Y., Zhu, Q., Jiang, J., Wu, C., Shen, T., 2016. Gestational and lactational exposure to low-dose bisphenol A increases Th17 cells in mice offspring. Environ. Toxicol. Pharmacol. 47,149158. doi: 10.1016/j.etap.2016.09.017.

Malaisé, Y., Ménard, S., Cartier, C., Lencina, C., Sommer, C., Gaultier, E., Houdeau, E., GuzylackPiriou, L., 2018. Consequences of bisphenol a perinatal exposure on immune responses and gut barrier function in mice. Arch. Toxicol. 92.347-358. doi: 10.1007/s00204-017-2038-2.

Martinez, F.O., Helming, L., Gordon, S., 2009. Alternative activation of macrophages: an immunologic functional perspective. Annu. Rev. Immunol. 27,451-483. doi:

10.1146/annurev.immunol.021908.132532.

McNelis, J.C., Olefsky, J.M., 2014. Macrophages, immunity, and metabolic disease. Immunity. 41,3648. doi: 10.1016/j.immuni.2014.05.010.

McWhorter, F.Y., Wang, T., Nguyen, P., Chung, T., Liu, W.F., 2013. Modulation of macrophage phenotype by cell shape. Proc. Natl. Acad. Sci. U S A. 110, 17253-17258. doi:

10.1073/pnas.1308887110.

Murray, P.J., Allen, J.E., Biswas, S.K., Fisher, E.A., Gilroy, D.W., Goerdt, S., Gordon, S., Hamilton, J.A., Ivashkiv, L.B., Lawrence, T., Locati, M., Mantovani, A., Martinez, F.O., Mege, J.L., Mosser, D.M., Natoli, G., Saeij, J.P., Schultze, J.L., Shirey, K.A., Sica, A., Suttles, J., Udalova, I., van Ginderachter, J.A., Vogel, S.N., Wynn, T.A.. 2014. Macrophage activation and polarization: nomenclature and experimental guidelines. Immunity. 41,14-20. doi: 10.1016/j.immuni.2014.06.008. 
Mustieles, V., Casas, M., Ferrando-Marco, P., Ocón-Hernández, O., Reina-Pérez, I., Rodríguez-

Carrillo, A., Vela-Soria, F., 2019. Bisphenol A and adiposity measures in peripubertal boys from the INMA-Granada cohort.Environ Res. 173, 443-451. doi: 10.1016/j.envres.2019.03.045.

Paun, A., Reinert, J.T., Jiang, Z., Medin, C., Balkhi, M.Y., 2008. Fitzgerald KA, Pitha PM.Functional characterization of murine interferon regulatory factor 5 (IRF-5) and its role in the innate antiviral response. J. Biol. Chem. 283,14295-14308. doi: 10.1074/jbc.M800501200.

Pfeifer, D., Chung, Y.M., Hu, M.C., 2015. Effects of Low-Dose Bisphenol A on DNA Damage and Proliferation of Breast Cells: The Role of c-Myc. Environ. Health Perspect. 123,1271-1279. doi: 10.1289/ehp.1409199.

Pyo, M.Y., Kim, H.J., Back, S.K., Yang, M., 2007. Downregulation of peritoneal macrophage activity in mice exposed to bisphenol A during pregnancy and lactation. Arch. Pharm. Res. 30,1476-1481. Rogers, J.A., Metz, L., Yong, V.W., 2013. Review: Endocrine disrupting chemicals and immune responses: a focus on bisphenol-A and its potential mechanisms. Mol. immunol. 53,421-430. doi: 10.1016/j.molimm.2012.09.013.

Rogers, J.A., Mishra, M.K., Hahn, J., Greene, C.J., Yates, R.M., Metz, L.M., Yong, V.W., 2017. Gestational bisphenol-A exposure lowers the threshold for autoimmunity in a model of multiple sclerosis. Proc. Natl. Acad. Sci. U S A. 114,4999-5004. doi: 10.1073/pnas.1620774114.

Satoh, T., Takeuchi, O., Vandenbon, A., Yasuda, K., Tanaka, Y., Kumagai, Y., Miyake, T., Matsushita, K., Okazaki, T., Saitoh, T., 2010. The Jmjd3-Irf4 axis regulates M2 macrophage polarization and host responses against helminth infection. Nat. Immunol. 11,936-944. doi: 10.1038/ni.1920.

Senyildiz, M., Karaman, E.F., Bas, S.S., Pirincci, P.A., Ozden, S., 2017. Effects of BPA on global DNA methylation and global histone 3 lysine modifications in SH-SY5Y cells: An epigenetic mechanism linking the regulation of chromatin modifiying genes. Toxicol. In Vitro. 44,313-321. doi: 10.1016/j.tiv.2017.07.028.

Sica, A., Mantovani, A., 2012. Macrophage plasticity and polarization: in vivo veritas. J. Clin. Invest. 122,787-795. doi: 10.1172/JCI59643.

Teixeira, D., Marques, C., Pestana, D., Faria, A., Norberto, S., Calhau, C., Monteiro, R., 2016. Effects of xenoestrogens in human M1 and M2 macrophage migration, cytokine release, and estrogen-related signaling pathways.Environ. Toxicol. 31,1496-1509. doi: 10.1002/tox.22154. 
Vandenberg, L.N., Chahoud, I., Heindel, J.J., Padmanabhan, V., Paumgartten, F.J., 2010. Schoenfelder

G. Urinary, circulating, and tissue biomonitoring studies indicate widespread exposure to bisphenol A. Environ. Health Perspect. 118,1055-1070. doi: 10.1289/ehp.0901716.

Wassenaar, P.N.H., Trasande, L., Legler, J., 2017. Systematic review and Meta-analysis of early-life exposure to Bisphenol A and obesity-related outcomes in Rodents. Environ. Health Perspect.

125,106001. doi: 10.1289/EHP1233.

Weiss, M., Byrne, A.J., Blazek, K., Saliba, D.G., 2015. Pease JE, Perocheau D, Feldmann M, Udalova IA. IRF5 controls both acute and chronic inflammation. Proc. Natl. Acad. Sci. U S A. 112,1100111006. doi: 10.1073/pnas.1506254112.

Wynn, T.A., Chawla, A., Pollard, J.W., 2013. Macrophage biology in development, homeostasis and disease. Nature. 496,445-455. doi: 10.1038/nature12034.

Yanai, H., Chen, H.M., Inuzuka, T., Kondo, S., Mak, T.W., 2007. Takaoka A, Honda K, Taniguchi T. Role of IFN regulatory factor 5 transcription factor in antiviral immunity and tumor suppression. Proc. Natl. Acad. Sci. U S A. 104,3402-3407. doi: 10.1073/pnas.0611559104.

Zbucka-Kretowska, M., Poplawska, I., Kretowska, A., Moniuszko, M., Grubczak, K., 2017. Short-term in vitro effects of bisphenol A activity on phenotype and function of peripheral blood immune system cells. Food Chem. Toxicol. 110,262-273.doi: 10.1016/j.fct.2017.10.034. 


\section{Legends}

Fig.1 Morphological changes of mouse peritoneal macrophages after $24 \mathrm{~h}$ of polarization (Scale bars, $200 \mu \mathrm{m}$.)

A. Image of - unpolarized macrophages. B. Image of polarized macrophages in M1 type

conditions of polarization after 24h. C. Image of pPolarized macrophages in M2 type conditions of polarization after 24h. D. Quantification of cell area for three macrophage populations. E.

Quantification of cell elongation factor for three macrophage populations. The results are presented as the mean \pm S.E.M. In the same unit area, the cell number were 27,48 and 46 for unpolarized macrophages, polarized macrophages under M1 type conditions and polarized macrophages under M2 type conditions, respectively. One way ANOVA with Tukey post hoc test was used to compare groups. ${ }^{*} \mathrm{p}<0.05$ and ${ }^{* *} \mathrm{p}<0.01$ compared with the unpolarized counterpart.

Fig.2 Effects of BPA exposure on polarization of mouse peritoneal macrophages in M1 type condition

A. Representative scatter plot of CD11c positive cells analyzed by FCM with FITC-CD11c. B. The number of CD11c positive cells by FCM analysis. C. Relative mRNA expression of the surface marker CD11c quantified by qRT-PCR. D. Activity of functional marker iNOS quantified by ELISA. E. Relative mRNA expression of the functional marker iNOS quantified by qRT-PCR. The results are presented as the mean \pm S.E.M. from independent experiments in each group $(\mathrm{n}=3)$. One way ANOVA with Tukey post hoc test was used to compare groups. ${ }^{*} \mathrm{p}<0.05$ and ${ }^{* *} \mathrm{p}<0.01$ compared with the control counterpart.

Fig.3 Effects of BPA exposure on polarization of mouse peritoneal macrophages in M2 type condition

A. Representative scatter plot of CD206 positive cells analyzed by FCM with FITC-CD206. B. The number of CD206 positive cells by FCM analysis. C. Relative mRNA expression of surface marker CD206 quantified by qRT-PCR. D. Activity of functional marker Arg-1 quantified by ELISA. E. Relative mRNA expression of functional marker Arg-1 quantified by qRT-PCR. The results are presented as the mean \pm S.E.M. from independent experiments in each group $(n=3)$. 
One way ANOVA with Tukey post hoc test was used to compare groups. ${ }^{*} \mathrm{p}<0.05$ and ${ }^{* *} \mathrm{p}<$ 0.01 compared with control counterpart.

Fig.4 Effect of BPA exposure on the expression of cytokines secreted by M1 subtype in M1 type condition. The contents of TNF- $\alpha$ (A), IL-6 (C), MCP-1(E) in supernatants were quantified by ELISA. Relative mRNA expressions of TNF- $\alpha$ (B), IL-6 (D), MCP-1(F) were quantified by qRTPCR. The results are presented as the mean \pm S.E.M. from independent experiments in each group $(\mathrm{n}=3)$. One way ANOVA with Tukey post hoc test was used to compare groups. ${ }^{*} \mathrm{p}<0.05$ and $* * \mathrm{p}<0.01$ compared with the control counterpart.

Fig.5 Effect of BPA exposure on the expression of cytokines secreted by M2 subtype in M2 type condition. The contents of IL-10 (A) and TGF- $\beta$ (C) in supernatants were quantified by ELISA. Relative mRNA expressions of IL-10 (B) and TGF- $\beta$ (D) were quantified by qRT-PCR. The results are presented as the mean \pm S.E.M. from independent experiments in each group $(n=3)$. One way ANOVA with Tukey post hoc test was used to compare groups. $* \mathrm{p}<0.05$ and $* * \mathrm{p}<$ 0.01 compared with the control counterpart.

Fig.6 Effect of BPA exposure on the expression of IRF5 and IRF4 in M1 condtion of polarization A. Relative mRNA expression was quantified by qRT-PCR. B. Protein expression was analyzed by Western blot, b-Actin served as the loading control. The results are presented as the mean \pm S.E.M. from independent experiments in each group $(\mathrm{n}=3)$. One way ANOVA with Tukey post hoc test was used to compare groups. ${ }^{*} \mathrm{p}<0.05$ and ${ }^{* *} \mathrm{p}<0.01$ compared with the control counterpart.

Fig.7 Effect of BPA exposure on the expression of IRF5 and IRF4 in M2 condition polarization.

A. Relative mRNA expression was quantified by qRT-PCR. B. Protein expression was analyzed by Western blot, $\beta$-Actin served as the loading control. The results are presented as the meant S.E.M. from independent experiments in each group $(\mathrm{n}=3)$. One way ANOVA with Tukeypost hoc test was used to compare groups. 
Fig.1
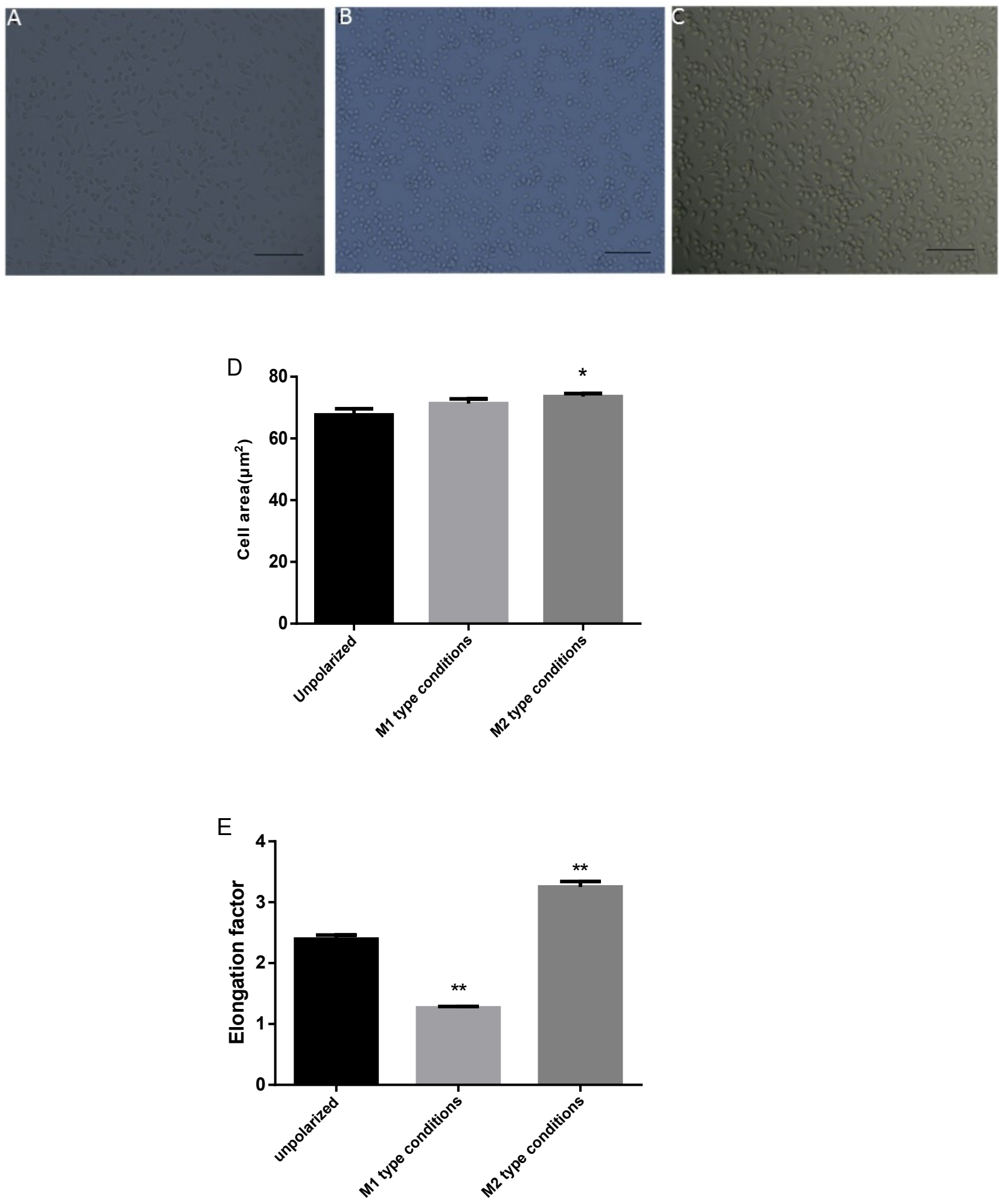
Fig.2

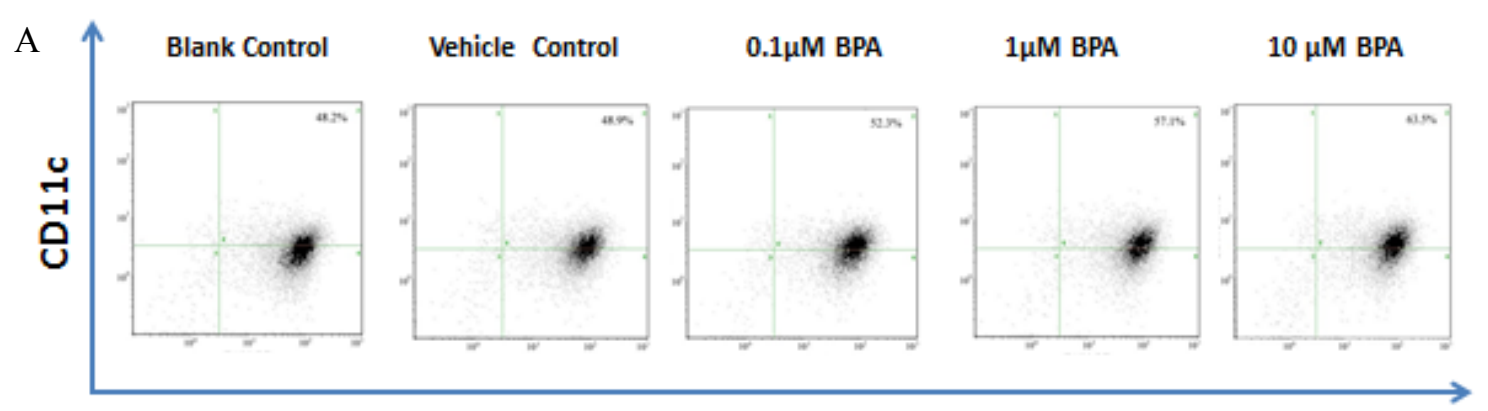

F4/80

B

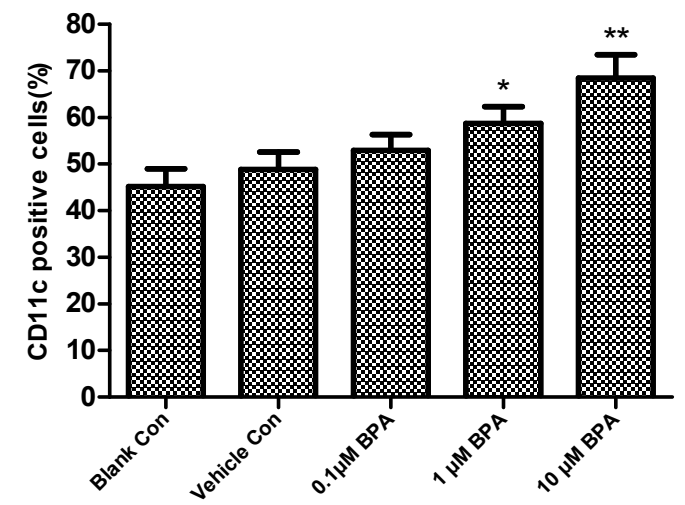

D

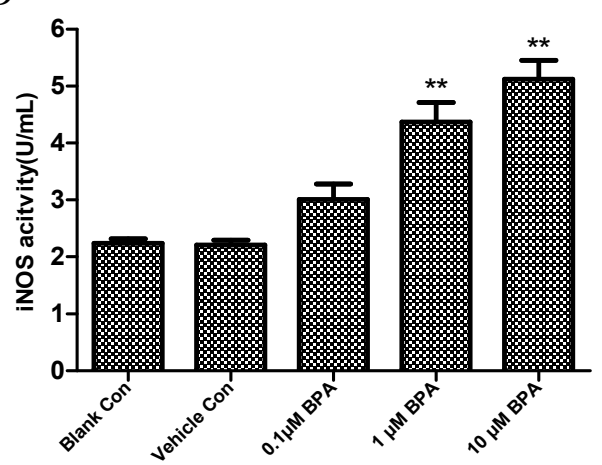

C

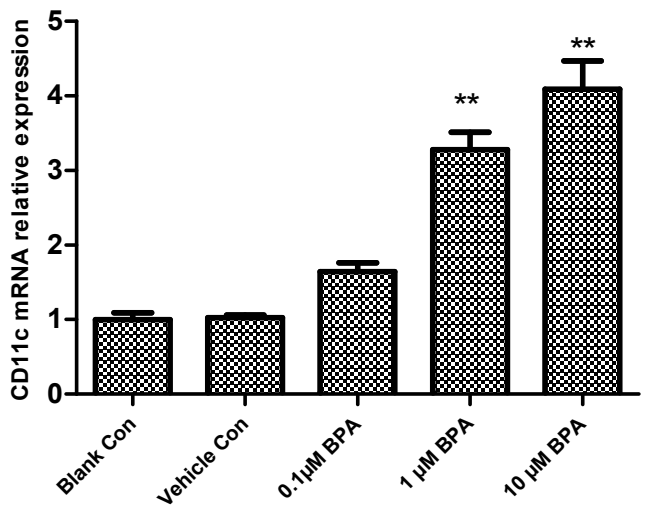

E

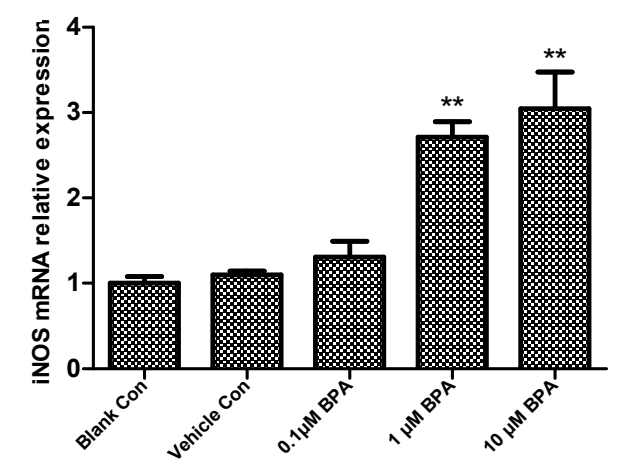


Fig.3

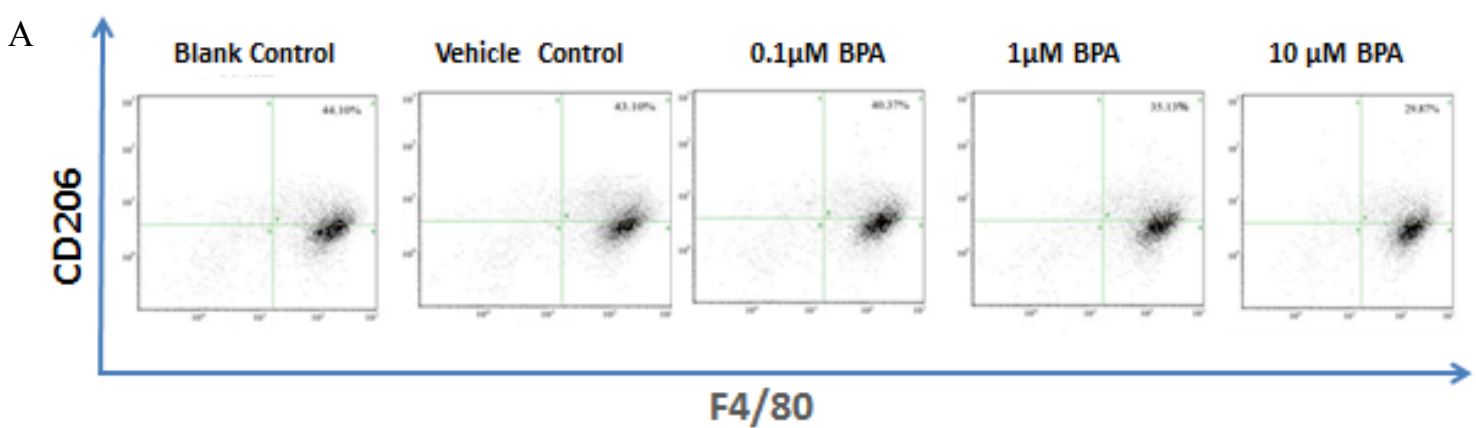

B
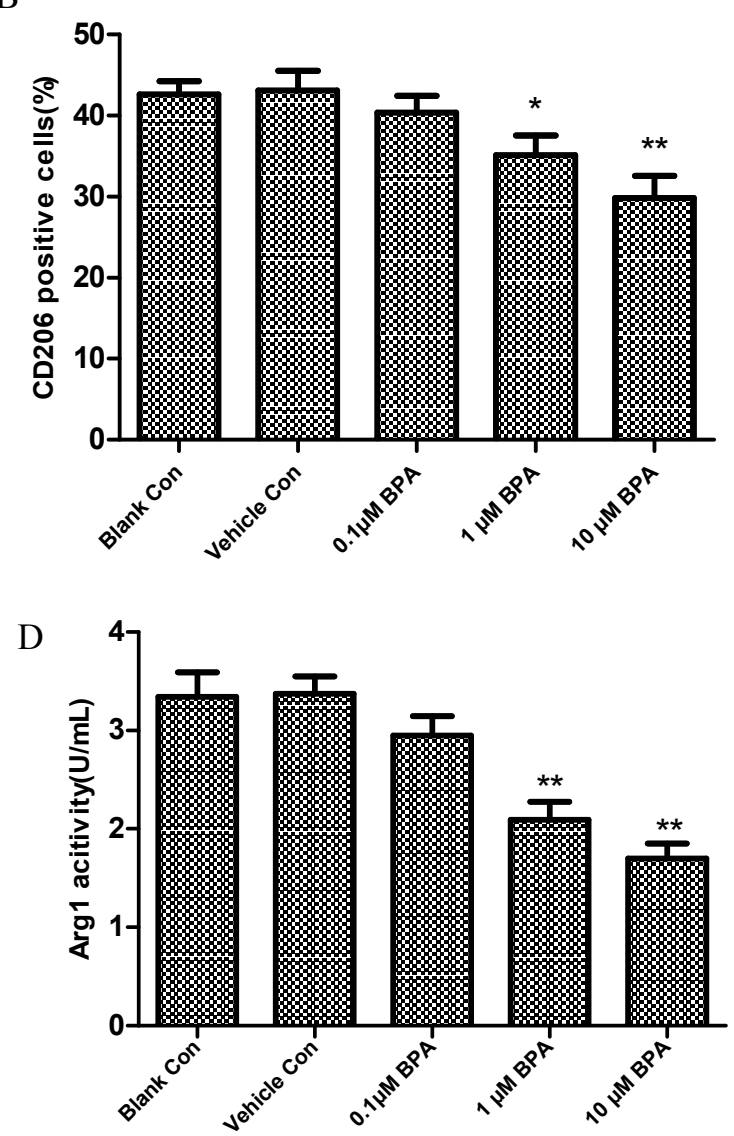

C
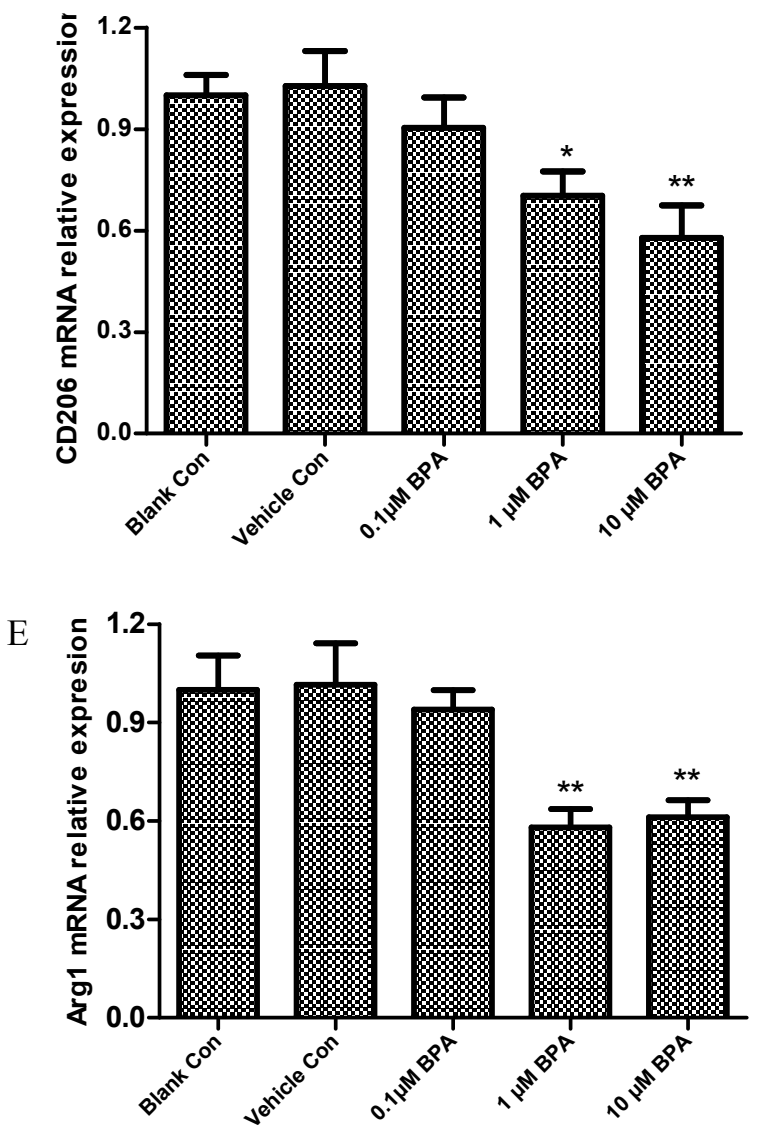
Fig.4
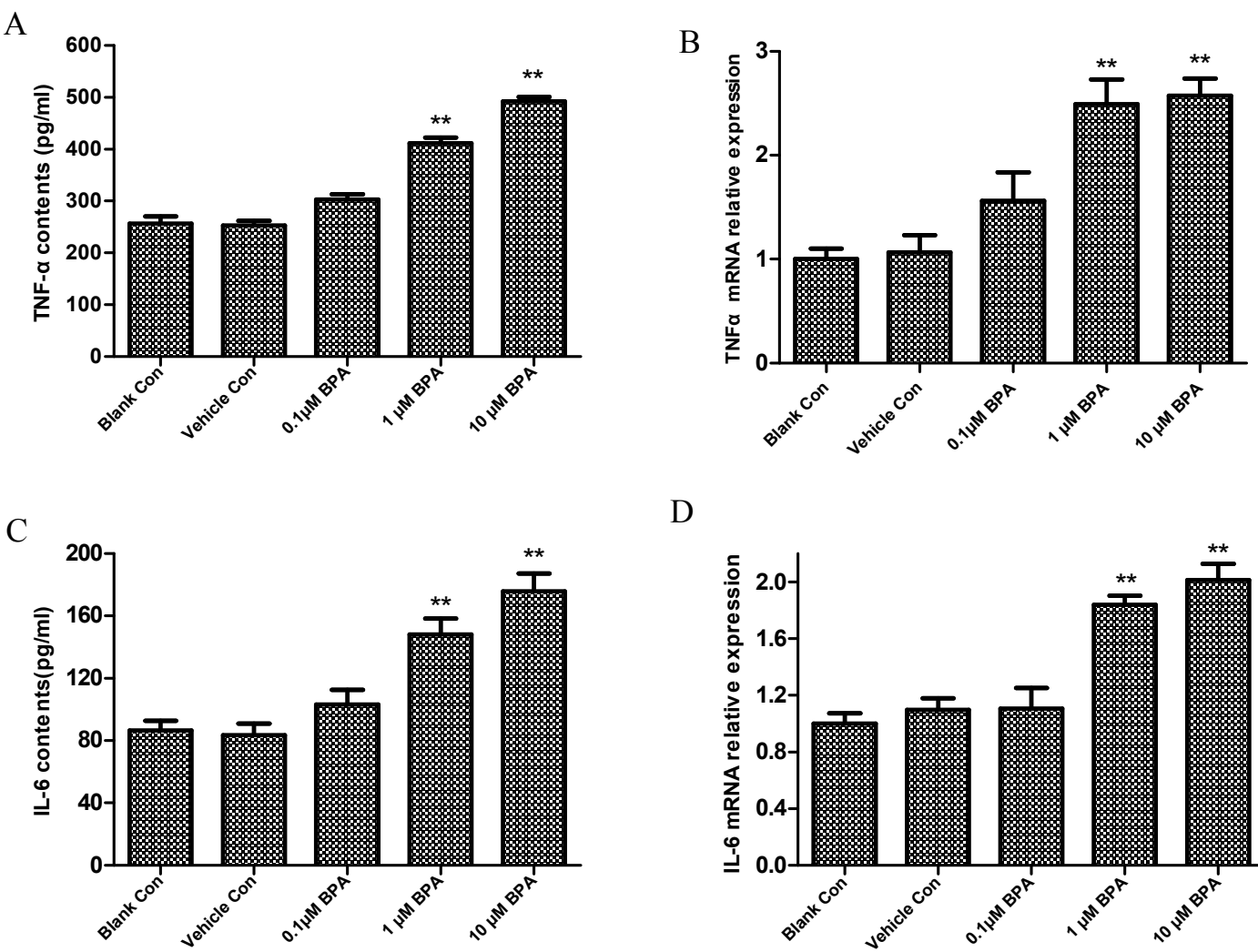

D

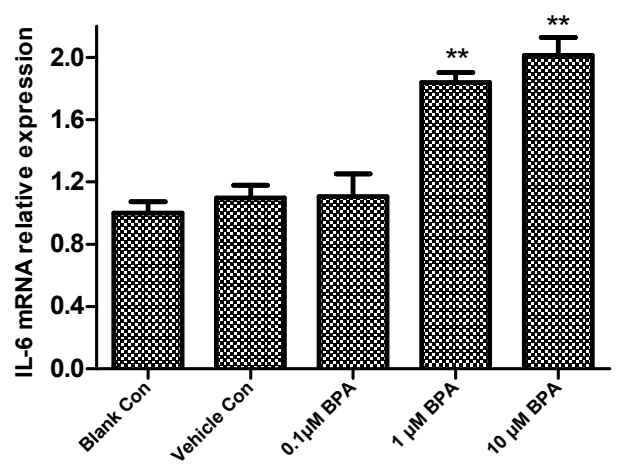

E

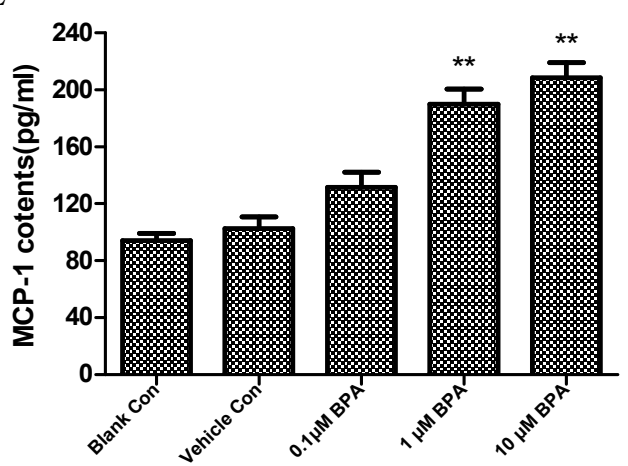

F

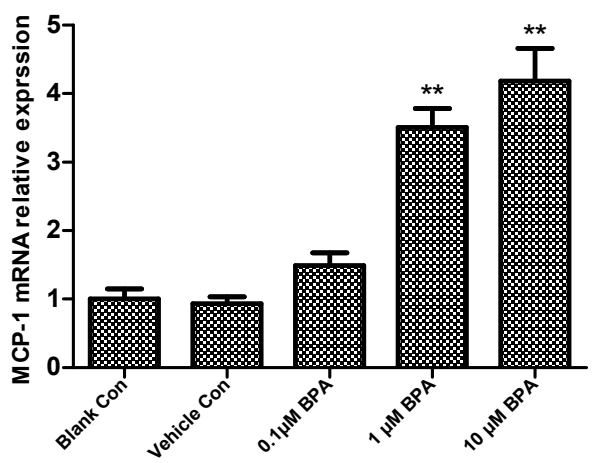


Fig. 5
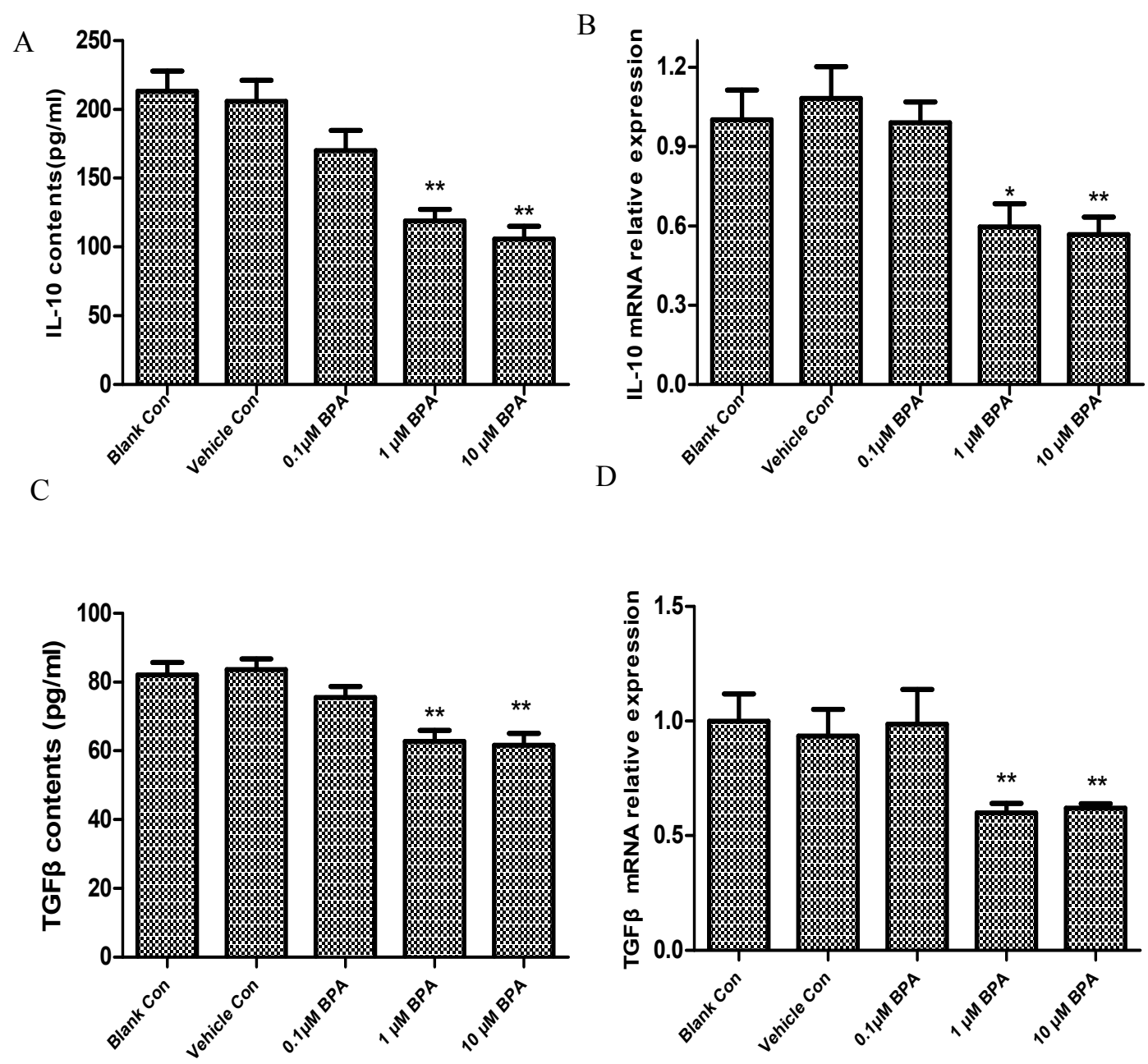
Fig.6

A

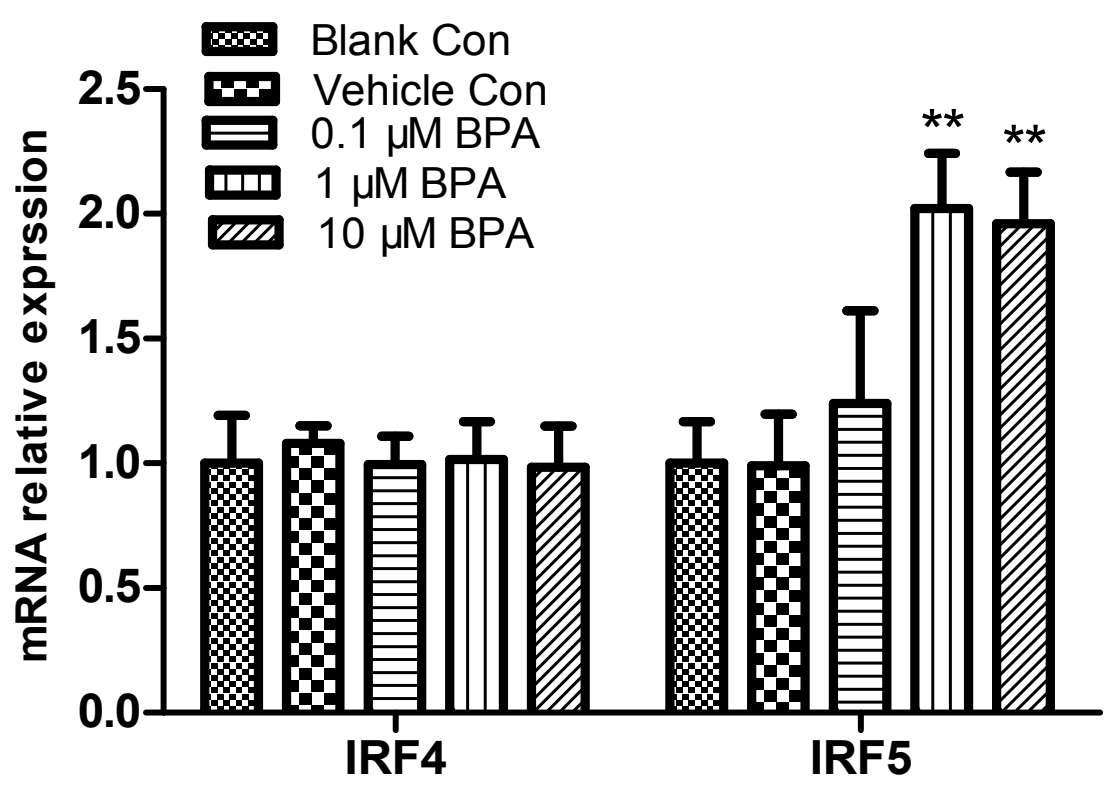

B

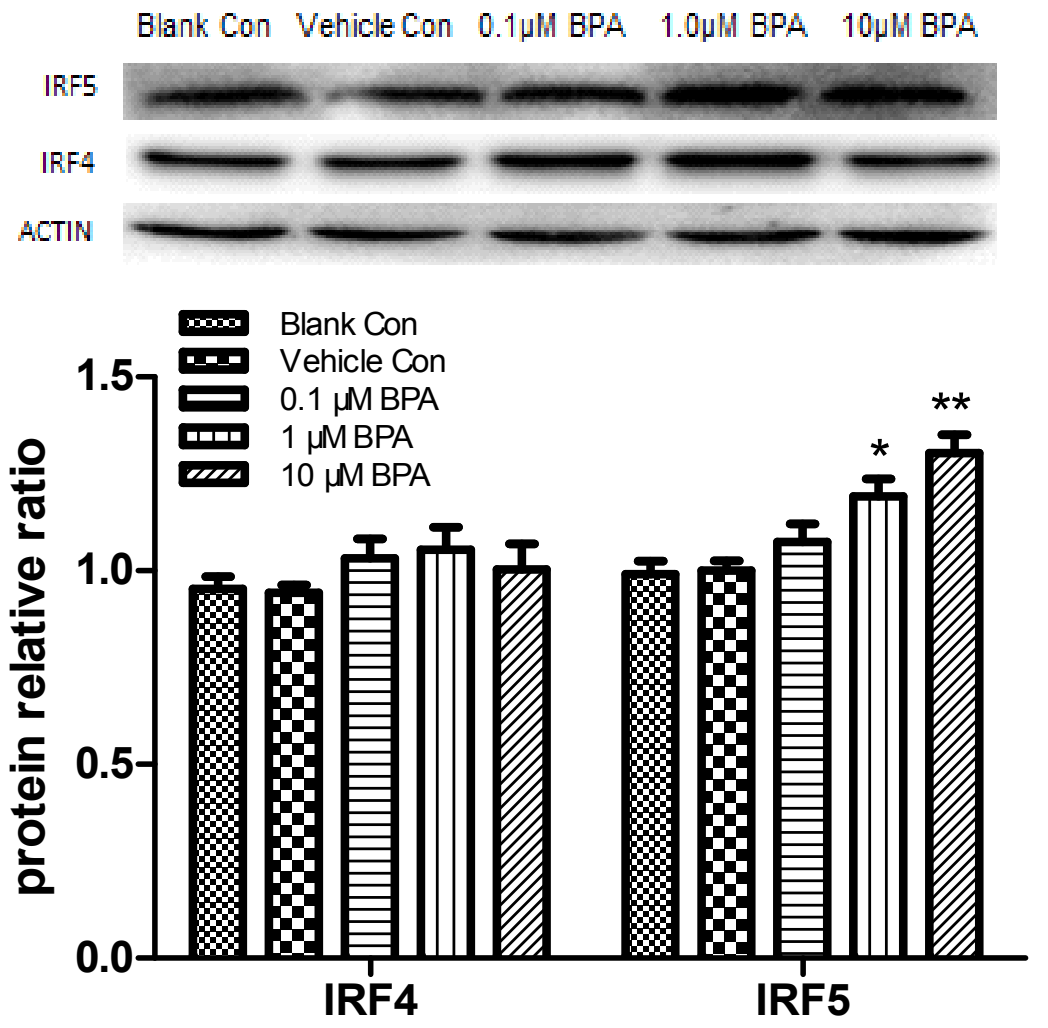


Fig.7

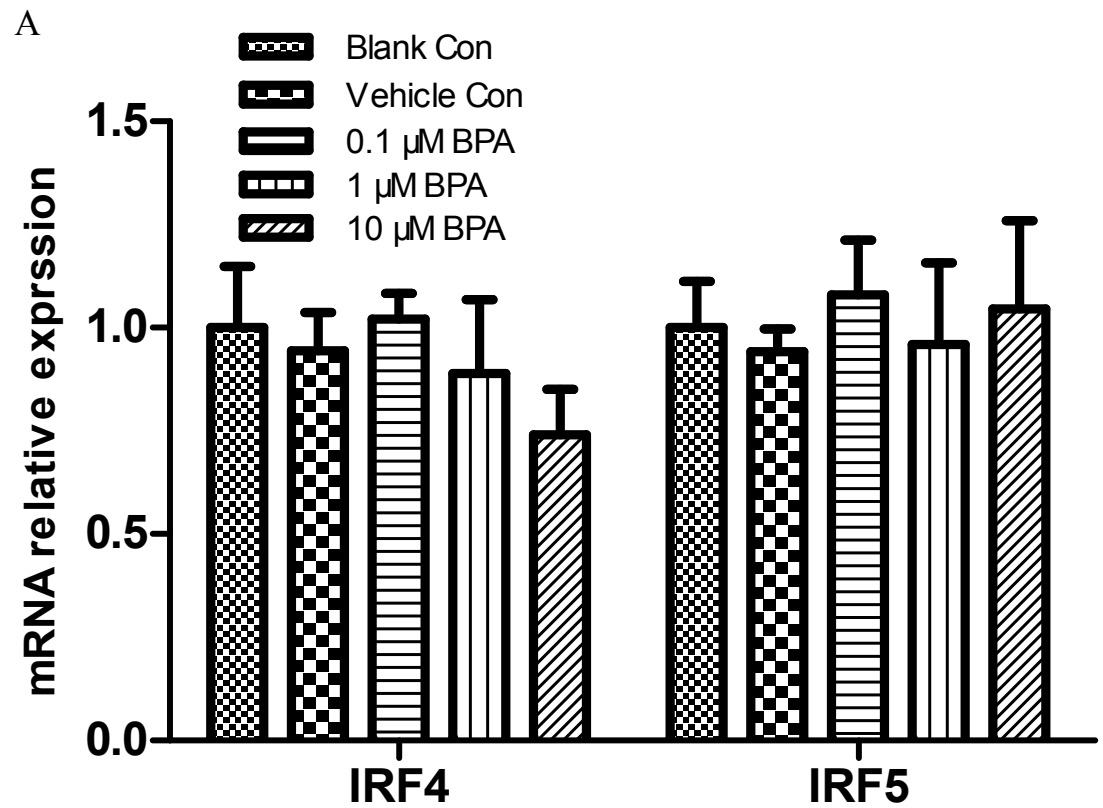

B
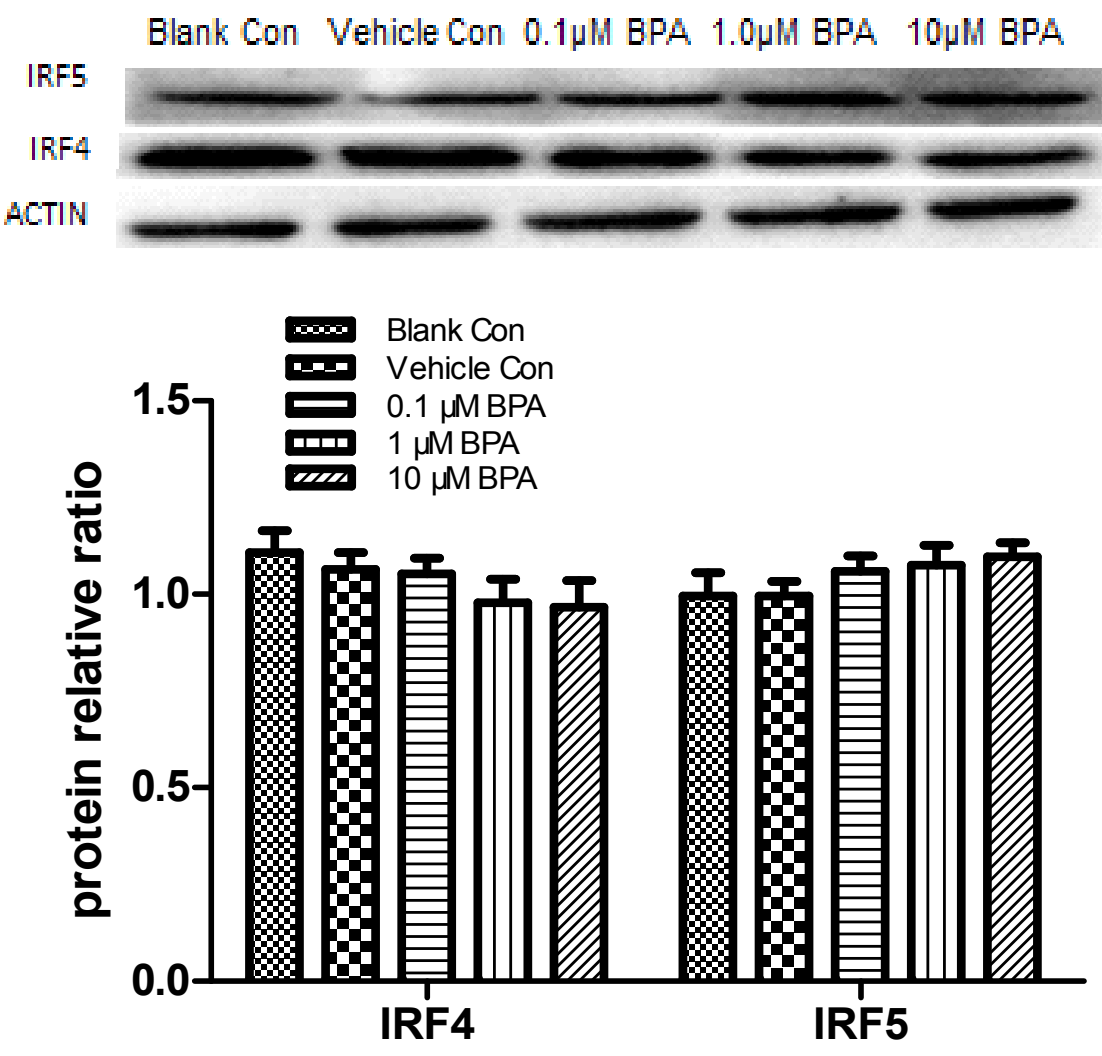
Table 1 Effects of 24h polarization on cell number, activities of iNOS or Arg-1 in M1 or M2 type conditions of polarization $(\bar{x} \pm s \mathrm{~d}, \mathrm{n}=3)$

\begin{tabular}{lcccc}
\hline & \multicolumn{2}{c}{ M1 type conditions of polarization } & \multicolumn{2}{c}{ M2 type conditions of polarization } \\
\hline & number of M1 (\%) & iNOS activity (U/L) & number of M2 (\%) & Arg-1activity (U/L) \\
unpolarized & $19.27 \pm 3.46$ & $0.45 \pm 0.05$ & $18.33 \pm 3.85$ & $0.42 \pm 0.05$ \\
24h of polarized & $48.23 \pm 5.16^{* *}$ & $2.36 \pm 0.12^{* *}$ & $42.63 \pm 2.81^{* *}$ & $3.34 \pm 0.43^{* *}$ \\
\hline
\end{tabular}

${ }^{* *} P<0.01$ versus unpolarized counterparts. 